Med Klin Intensivmed $2011 \cdot 106: 5-5$ DOI 10.1007/s00063-011-0002-0

Online publiziert: 22. September 2011

(c) Springer-Verlag 2011

\author{
M. Buerke ${ }^{1} \cdot$ A. Gasser ${ }^{2} \cdot$ T. Suhai ${ }^{2}$ \\ ${ }^{1}$ Zentrum für Innere Medizin, Universitätsklinik und Poliklinik für Innere \\ Medizin III, Martin-Luther-Universität Halle-Wittenberg, Halle (Saale) \\ 2 Springer-Verlag GmbH, Heidelberg
}

\section{Medizinische Klinik - Intensivmedizin und Notfallmedizin}

schrift mit wissenschaftlichem Anspruch ändert sich durch die Fusion der beiden Titel nichts. Die im Jahre 2009 eingeführten Leitthemen mit Übersichtsbeiträgen zu aktuellen Fragestellungen der gesamten Intensiv- und Notfallmedizin, die Zertifizierte Fortbildung - CME und die verschiedenen Rubriken (Originalien, Kasuistiken, Für Sie gelesen, Innovationen in der Intensivmedizin, u. a.) bleiben in ihrer jetzigen Form erhalten. Wie die Ende vergangenen Jahres durchgeführte Leserbefragung zeigte, wird dieses Konzept sowohl von den Autoren als auch von den Lesern sehr gut angenommen.

\section{? Nutzen Sie beide Online-Archive!}

Dem Wesen der Intensivmedizin entsprechend war und ist die Zeitschrift interdisziplinär angelegt und lebt von der Vielgestaltigkeit ihrer Themen. Für die Zukunft erhoffen wir uns, die Zahl der frei eingereichten Arbeiten weiter zu steigern und die Zeitschrift damit für unsere Leser noch attraktiver gestalten zu können. Unterstützen Sie die Medizinische KlinikIntensivmedizin und Notfallmedizin bitte auch weiterhin, indem Sie sie als Ihr Publikationsorgan nutzen und fördern.

Als Abonnent von Medizinische KlinikIntensivmedizin und Notfallmedizin haben Sie künftig unter www.intensivmedizin. springer.de (Button „Read online“) Zugriff auf beide Online-Archive, auf das Archiv der Intensivmedizin und Notfallmedizin und das Archiv der Medizinische KlinikIntensivmedizin und Notfallmedizin, das auch die früheren Jahrgänge der Medizinischen Klinik enthält.

Ihr

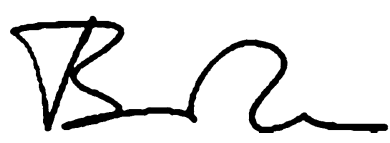

Prof. Dr. Michael Buerke

Medizinische Klinik -

Intensivmedizin und Notfallmedizin

Ihre

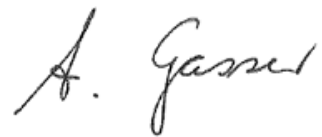

Dr. Annette Gasser

Redaktionsleitung Medizinische Klinik Intensivmedizin und Notfallmedizin

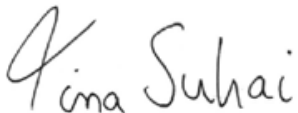

Dr. Tina Suhai

Redaktion Medizinische Klinik Intensivmedizin und Notfallmedizin
Koordinierender Herausgeber
An dem erfolgreichen Konzept der Intensivmedizin und Notfallmedizin als deutschsprachiger Fortbildungszeit- 